\title{
Criou Fama, Agora Deita na Cama! Construção de uma Escala de Reputação de Restaurantes
}

\author{
Created Fame, Now Lie on the Bed! Construction of a Restaurant Reputation Scale \\ Has Creado Fama, ¡Ahora Acuéstate en la Cama! Construcción de una Escala de Reputación \\ de Restaurantes
}

\author{
Luana de Oliveira Alves ${ }^{1}$ \\ Viviane Santos Salazar ${ }^{2}$ \\ Indira Gandhi Bezerra-de-Sousa ${ }^{3}$
}

\begin{abstract}
Resumo
Os restaurantes de alta gastronomia estão inseridos em um setor em que a reputação é um atributo importante de escolha dos clientes. Embora a reputação seja um recurso intangível, fundamentado na Visão Baseada em Recursos (VBR), pode ser mensurado, conforme apontam vários estudos anteriores. Portanto, o presente artigo objetivou construir uma escala de mensuração da reputação a partir da percepção de gestores de restaurantes de alta gastronomia. Como um estudo de natureza metodológica, a construção da escala ocorreu em sete etapas, com destaque para a realização de treze entrevistas semiestruturadas, que resultaram na geração de itens do questionário. Em seguida, realizou-se a validação de face e conteúdo com cinco especialistas. A aplicação do questionário foi feita por meio de um link enviado por e-mail a gestores de restaurantes, finalizando com 65 respostas válidas. Inicialmente, a escala gerou 57 itens, que, após a purificação, foram reduzidos para 24, distribuídos em sete dimensões. A construção da escala gerou duas dimensões antes não contempladas na literatura: a qualidade do atendimento e a força das mídias sociais. No campo teórico, foi possível ampliar o número de estudos que buscam superar a dificuldade de se mensurar recursos intangíveis, bem como o desenvolvimento de pesquisas em um setor carente, encorajando novas investigações. No campo prático, a escala representa uma ferramenta de diagnóstico organizacional para intervenções em restaurantes gastronômicos, mas também pode ser utilizada em qualquer outro tipo, além do setor de serviços em geral.
\end{abstract}

Palavras-chaves: Reputação. Visão Baseada em Recursos (RBV). Restaurantes.

\begin{abstract}
Haute cuisine restaurants are inserted in an industry where reputation is an important attribute of choice for customers. Although reputation is an intangible resource, based on the Resource-Based View (VBR), it can be measured, as indicated by several previous studies. Therefore, this article aimed to build a reputation measurement scale based on the perception of haute cuisine restaurants. As a methodological study, the construction of the scale occurred in seven stages, with emphasis on thirteen semi-structured interviews, which resulted in the generation of questionnaire items. Then, the face and content validation were carried out with five specialists. The questionnaire was applied through a link sent by e-mail to restaurant managers, ending with 65 valid responses. Initially, the scale generated 57 items, which, after purification, were reduced to 24 , distributed in seven dimensions. The construction of the scale generated two dimensions not previously covered in the literature: the quality of service and the strength of social media. In the theoretical field, it was possible to increase the number of studies that seek to overcome the difficulty of measuring intangible resources, as well as the development of researches in a needy sector, encouraging new investigations. In the practical field, the scale represents an organizational
\end{abstract}

Doutoranda em administração pela UFPE/PROPAD. Mestre em administração pela UFPE/PROPAD. Bacharelado em Administração pela UESPI em 2013.

9 Doutora em administração pela UFPE. Mestre em Administração pela UFPE/PROPAD. Professora de pós-graduação em administração, Hotelería e Turismo na UFPE.

9 Doutoranda em Administração na Universidade Federal do Paraná (UFPR). Professora de Administração da Universidade Estadual do Piauí (UESPI). 
diagnostic tool for interventions in gastronomic restaurants, but it can also be used in any other type, besides the service sector in general.

Keywords: Reputation. Resource Based View (RBV). Restaurant.

\section{Resumen}

Los restaurantes de alta gastronomía están inseridos en un sector en que la reputación es una importante calidad de la elección de los clientes. Aunque la reputación sea un recurso intangible, basado en la Visión Basada en Recursos (VBR), puede ser medido, según varios estudios anteriores. Por tanto, el presente trabajo objetivó construir una escala de medición de la reputación a partir de la percepción de gestores de restaurantes de alta gastronomía. Como un estudio de naturaleza metodológica, la construcción de la escala ocurrió en siete etapas, con enfoque para la realización de trece entrevistas semi estructuradas, que resultaron en la creación de los elementos del cuestionario. En seguida, se realizó la convalidación de cara y contenido con cinco especialistas. La aplicación del cuestionario se hizo por medio de un link enviado por correo electrónico 65 respuestas válidas. De inicio, la escala creó 57 elementos, que, después de la purificación, fueron reducidos para 24, repartidos en siete dimensiones. La construcción de la escala origino dos dimensiones no contempladas antes en la literatura: la calidad del atendimiento y la fuerza de las redes sociales. En el campo teórico, fue posible ampliar el número de estudios que buscan superar la dificultad de medir recursos intangibles, así como el desarrollo de investigaciones en un sector carente, fomentando nuevas investigaciones. En el campo práctico, la escala representa una herramienta de diagnóstico organizacional para intervenciones en restaurantes gastronómicos, pero también se puede utilizar en otro tipo cualquiera, además del sector de servicios en general.

Palabras clave: Reputación; Visión Basada en Recursos (RBV); Restaurantes.

\section{Introdução}

O papel dos restaurantes na sociedade consiste, especialmente, em proporcionar boas experiências ao consumidor, indo além da venda de produtos/serviços (HANEFORS; MOSSBERG, 2003). De fato, "comer fora" tornou-se um hábito frequente entre os consumidores na atualidade, pois, devido às mudanças na economia e na estrutura familiar, foi possível que mais pessoas vivessem essa experiência. Segundo a Associação Brasileira de Bares e Restaurantes, cerca de $30 \%$ dos gastos do brasileiro com alimentação são destinados a refeições realizadas em bares e restaurantes, mas, de acordo com essa mesma pesquisa, a tendência é que, até 2020, esses gastos cresçam, chegando a quase $50 \%$. Esse crescimento está relacionado a vários fatores, como o aumento do número de escolas nacionais de gastronomia e do número de restaurantes e programas de televisão com o tema de gastronomia, e a transição de restaurantes mais simples, com cardápios modestos e sem originalidade, a uma profusão de restaurantes gastronômicos, também denominados de alta gastronomia, que são caracterizados, principalmente, pela presença de um chef renomado (FONSECA, 2000), competindo no mercado (SALAZAR, 2015).

Um importante fator que tem chamado atenção nesse tipo de restaurante é a reputação, apontada como fator primordial que, direta ou indiretamente, influencia quase todos os aspectos do trabalho gastronômico (STIERAND; DORFLER, 2012).

A reputação organizacional é um fenômeno amplamente discutido na atualidade. $O$ interesse de pesquisadores pela temática iniciou-se na década de 90 (GOTSI; WILSON, 2001) e houve contribuições de diversos campos de estudo economia, contabilidade, marketing, comunicação, teoria organizacional, sociologia e estratégia (FOMBRUM; GARDBERG; SEVER, 2000). Especificamente no campo da estratégia, foco deste trabalho, a reputação se configura como um recurso intangível difícil de replicar (DIERICKX; COOL, 1989). Com forte apoio teórico da Visão Baseada em Recursos (RBV), a literatura (DIERICKX; COOL, 1989; HALL, 1992; 1993) tem destacado sua relevância não apenas entre os clientes, mas também entre os fornecedores (BARNEY, 1991) e diversos outros grupos de stakeholders (GOTSI; WILSON, 2001).

A perspectiva da RBV trouxe uma nova luz sobre as opções estratégicas, porque envolve a exploração e desenvolvimento de novos recursos. Contudo, conforme destaca Molloy et al. (2011), a RBV ainda necessita avançar nas pesquisas empíricas acerca dos recursos intangíveis, tendo em vista que esses recursos ainda não são facilmente observados ou medidos. Para contornar tal problema, Godfrey e Hill (1995) sugeriram que os estudiosos se concentrassem nas variáveis "observáveis", que determinam o grau de inobservabilidade de um recurso. Portanto, para mensurar esses tipos de recursos (variáveis abstratas e intangíveis), como reputação, o desenvolvimento de escalas é o meio mais propício e desafiador (COSTA, 2011).

Alguns trabalhos, como os de Michalisin, Smith e Kline (1997), Hall (1992; 1993), Fombrun, Gardberg e Sever (2000) e Thomaz e Brito (2010), mensuraram a reputação considerando organizações de distintos setores, contudo as formas consideradas para mensurar tal recurso podem variar de acordo com a indústria, mercado, localização geográfica e recursos humanos disponíveis, surgindo a necessidade de que os indicadores de mensuração contribuam para que essas medidas sejam plenamente compreendidas e relevantes à indústria a que se deseja pesquisar (SMITH, 2008). 
Além disso, Walker (2010) pontua que, do ponto de vista da operacionalização, os pesquisadores não podem medir a reputação a partir da percepção agregada de todas as partes interessadas em um único artigo. Portanto, o autor sugere que estudos, em primeiro lugar, especifiquem as partes interessadas e os elementos analisados para poder medi-la.

A partir de tais considerações, o objetivo deste estudo consiste em construir uma escala de mensuração da reputação a partir da percepção dos gestores de restaurantes de alta gastronomia. Esta pesquisa contribui, em termos práticos, com uma ferramenta gerencial para os gestores de restaurantes gastronômicos, além de outros tipos de restaurantes e do setor de serviços em geral poderem utilizar como instrumento de autoavaliação. Em termos teóricos, este trabalho investigou uma lacuna de pesquisa ainda carente de estudos, contribuindo para o avanço da literatura de RBV ao criar uma maneira de tangibilizar um recurso intangível.

\section{Reputação Organizacional}

Para que a reputação da empresa se torne um recurso valioso, é fundamental que seja reconhecida positivamente, pois a presença de tal recurso permite à empresa conquistar clientes (MICHALISIN; KLINE; SMITH, 1997), cobrar preços premium (FOMBRUN; SHANLEY, 1990), atrair e reter funcionários, bem como atrair investidores (SMITH, 2008). No entanto, para que isso aconteça de fato, as atividades de uma organização devem alinhar-se com sua boa reputação, com fins a elevar a satisfação do cliente (O'ROURKE, 2004).

Os primeiros estudos de reputação concentravam-se no conceito de imagem corporativa, resultando em conflitos terminológicos e ambiguidades por serem termos apontados (imagem e reputação) como sinônimos. Assim, Gotsi e Wilson (2001) dividiram a literatura em duas escolas de pensamento dominante. A escola de pensamento análoga, que considera a reputação corporativa como sinônimo de imagem corporativa, e a escola de pensamento diferenciado, que considera os termos sob três pontos de vistas diferentes. A primeira entende que os conceitos são totalmente diferentes, enquanto que a segunda visão percebe a imagem como relacionada à reputação e, por fim, a terceira visão considera a reputação influenciada pelas múltiplas imagens das várias audiências, de modo que os conceitos estariam inter-relacionados, sendo essa a visão mais aceita entre os diversos pesquisadores e que, portanto, foi considerada neste trabalho.

Dessa forma, pode-se dizer que a reputação é um conceito coletivo construído a partir das percepções de vários stakeholders sobre o desempenho de uma dada organização (FOMBRUM; GARDBERG; SEVER, 2000). Neste trabalho, a medição da reputação será avaliada por um único grupo; no caso, a visão dos gestores. Na mensuração, levou-se em consideração as expectativas sociais dos gestores, ou seja, o que eles esperam como resultados da empresa, refletidos basicamente em qualidade de produtos e serviços, liderança na indústria, bom empregador, responsabilidade social e competência para os negócios (BERENS; VAN RIEL, 2004).

Alguns trabalhos têm desenvolvido escalas para mensurar a reputação. Por exemplo, Fombrun, Gardberg e Sever (2000) desenvolveram o quociente da reputação (RQ) que tem sido utilizado em alguns estudos. A escala compreende 20 itens, que representam seis dimensões da reputação corporativa, a saber: 1) apelo emocional, 2) produtos e serviços, 3) visão e liderança, 4) ambiente de trabalho, 5) responsabilidade social e ambiental, e 6) desempenho financeiro. Vale destacar que a dimensão apelo emocional, que aborda aspectos como confiança, admiração e respeito, tem sido apontada como um antecedente ou uma consequência da reputação, e não como uma faceta de reputação (SARSTEDT; WILCZYNSKIC; MELEWAR, 2013).

Helm (2005) elaborou uma nova escala visando garantir que a definição de reputação capturaria suficientemente seu domínio de conteúdo. Com isso, identificou dez indicadores de reputação, a saber: 1) qualidade dos produtos, 2) compromisso com a proteção do ambiente, 3) sucesso corporativo, 4) tratamento dos empregados, 5) orientação para o cliente, 6) compromisso com as questões sociais, 7) valor para o preço dos produtos, 8) desempenho financeiro, 9) qualificação da gestão e 10) credibilidade das reclamações publicitárias.

Thomaz e Brito (2010), ao elaborar sua escala, consideram dois grupos de stakeholders: clientes e funcionários, concluindo que, embora cada grupo perceba de maneira diferente como a reputação é formada, acabam por Ihes atribuir a mesma importância. Numa abordagem diferente, Walsh e Beatty (2007) introduziram a escala da reputação baseada no cliente (CBR).

Aponta-se, portanto, para a multidimensionalidade do construto, isto é, a reputação é formada a partir de várias dimensões: qualidade do produto/serviço, liderança no segmento (FOMBRUN, GARDBERG; SEVER, 2000; THOMAZ; BRITO, 2010), orientação para o cliente (WALSH, BEATTY, 2007), tratamento dos empregados (HELM, 2005) e responsabilidade social e ambiental (SARSTEDT;WILCZYNSKI; MELEWAR, 2013).

A realização de estudos que permitam a construção de escalas para intensificar os resultados da reputação no contexto dos restaurantes é demandada na literatura. Além disso, observa-se que, nas publicações nacionais, evita-se desenvolver escalas, utilizando as consolidadas internacionalmente (COSTA, 2011), que, muitas vezes, apresentam tanto problemas de tradução como culturais, não sendo adequadas ao cenário brasileiro.

A identificação das dimensões encontradas na literatura será base para a determinação das dimensões definidas na etapa de desenvolvimento da escala a ser discutida na próxima sessão. 


\section{Processo De Desenvolvimento Da Escala}

Baseando-se na literatura sobre desenvolvimento de escala (CHURCHILL, 1979; ROSSITER, 2002; SPECTOR, 1992), optou-se por seguir a construção em sete passos: i) especificação do domínio do construto; ii) atividades de geração de itens e validação de face e conteúdo; iii) construção do instrumento de pesquisa; iv) atividade de amostragem; v) procedimentos de limpeza da escala; vi) análise de validade e confiabilidade da escala e vii) desenvolvimento de normas e recomendações de interpretação, que foram descritos adiante.

A escala aqui desenvolvida pode ser utilizada para todos os tipos de restaurantes, apesar de ter sido realizada em um segmento específico. Além disso, pode ser aplicada para outros tipos de serviços, com o respaldo de que deve ser adequada ao contexto trabalhado, logo poderão ser excluídos itens ou adicionado novos, caso haja necessidade.

\subsection{Especificação do domínio do construto}

A especificação inicia-se por uma definição apropriada para o entendimento dos limites do conceito. Reputação corporativa é a avaliação geral de uma empresa ao longo do tempo, ou em qualquer outra forma de comunicação e simbolismo que forneça informações sobre as ações da empresa e/ou uma comparação com as ações de outros líderes rivais (GOTSI, WILSON, 2001).

Para a verificação da dimensionalidade do construto, foi considerada, além da revisão da literatura, a análise exploratória a partir de treze entrevistas semiestruturadas (ponderando o critério de saturação), realizadas em agosto e setembro de 2017 , com duração média de $1 \mathrm{~h} 30 \mathrm{~min}$. As entrevistas foram transcritas e analisadas com ajuda do Microsoft Excel, e geraram 7 (sete) dimensões, conforme Quadro 1. Todos os entrevistados eram especialistas ligados ao setor da gastronomia, provenientes de diversos segmentos, entre eles: professores da área de turismo ou hotelaria (2), chefs de cozinha (2), consultores da área gastronômica (2) representante da Abrasel (1), jornalista da área de gastronomia (3), gestores de restaurantes (3). A maior parte dos entrevistados é da cidade do Recife por uma questão de acessibilidade. Utilizou-se para essa etapa um roteiro semiestruturado, dividido em três partes: levantamento de informações pessoais do respondente, informações sobre o construto e, por fim, informação adicional sobre algo que o respondente considerasse interessante sobre o setor.

Quadro 1. Dimensões da reputação

\begin{tabular}{|l|l|}
\hline \multicolumn{1}{|c|}{ Dimensões encontradas } & \multicolumn{1}{c|}{ Definição } \\
\hline Qualidade do produto & Indicação de um produto considerado de "boa" qualidade para atender ao que o cliente espera. \\
\hline Qualidade do atendimento & $\begin{array}{l}\text { Avaliação da qualidade do atendimento levando em conta o tempo, a gentileza e outras } \\
\text { características importantes para atender bem um cliente. }\end{array}$ \\
\hline Qualidade do ambiente & Avaliação do ambiente físico, se agradável e confortável para atender aos clientes. \\
\hline Força das mídias sociais & $\begin{array}{l}\text { Características das mídias que interferem na "boa imagem" da empresa para os antigos e } \\
\text { novos clientes. }\end{array}$ \\
\hline Imagem do chef & Características do chef de cozinha que interferem na "boa imagem" da empresa. \\
\hline Responsabilidade social e ambiental & Avaliação de como a empresa se preocupa com questões sociais e ambientais. \\
\hline Reconhecimento da marca/preço & Reconhecimento da empresa na comunidade em geral, bem como reconhecimento do cliente. \\
\hline
\end{tabular}

Fonte: Elaborado pelos autores (2017).

Observou-se uma similaridade ao comparar as dimensões identificadas na literatura com as geradas na entrevista. Algumas dimensões permaneceram com a mesma nomenclatura e outras foram renomeadas, por exemplo, a dimensão "liderança" no segmento foi alterada para "sucesso". As dimensões "tratamento dos empregados", "habilidade para atrair e manter talentos" e "empresa financeiramente forte", previstas na literatura, não surgiram nas entrevistas, portanto, não foram consideradas. A dimensão "força das mídias" não é apontada na literatura como um indicador de mensuração, no entanto surgiu nas entrevistas. Em relação a este último aspecto, contudo, tem sido cada vez mais frequente o destaque das mídias sociais como importante fator a se considerar para a reputação a exemplo dos estudos de Salazar (2015), Aragão et al. (2016) e Azevedo et al. (2016). A dimensão "qualidade do atendimento" surge como uma nova dimensão, já que em restaurantes o atendimento é uma função fundamental e pode ser similar à "qualidade do serviço", prevista por Thomaz e Brito (2010) e Sarstedt, Wilczynski e Melewar (2013).

\subsection{Atividades de geração de itens e validação de face e conteúdo}

Nesta etapa, a primeira decisão diz respeito à realização de entrevistas semiestruturadas com pessoas do setor para capturar melhor o domínio especificado dos construtos, conforme apontado por Churchill (1979). Convém 
ressaltar que as entrevistas utilizadas foram as mesmas que permitiram realizar o dimensionamento dos construtos na etapa anterior a esta (especificação do domínio do construto).

Após a análise individual de cada uma das entrevistas, foram comparadas e observadas quais palavras-chaves se repetiam e, a partir disso, os itens foram elaborados. Vale destacar que a geração de itens nas entrevistas foi comparada aos itens previstos na literatura, no intento de adequá-los ao contexto da pesquisa.

Foram gerados 57 itens, dividindo-os em sete dimensões. Para cada uma das dimensões foram gerados oito itens, exceto a dimensão "qualidade do atendimento", para a qual foram gerados nove itens. Após isso, os mesmos foram submetidos ao julgamento de cinco especialistas, entre eles, dois doutores e dois doutorandos da área de administração e um doutorando da área de turismo. Todos com experiência em construção de escalas. Cada avaliador considerou dois critérios: i) itens julgados como adequados, conforme a definição do construto; ii) observação e sugestões de outros itens, bem como as propostas para melhorar o enunciado dos itens. Como critério de exclusão, foi estabelecido que se dois ou mais especialistas julgassem como inadequado, o item seria excluído, além da verificação das sugestões e comentários de itens com o mesmo sentido. Dessa forma, foram eliminados 23 itens, portanto, 34 itens foram para a etapa seguinte.

\subsection{Construção do instrumento de pesquisa}

Quanto à elaboração do instrumento de pesquisa, os 34 itens foram dispostos aleatoriamente para evitar vieses nas respostas. $O$ instrumento utilizado nessa pesquisa foi dividido da seguinte forma: i) Bloco I - perguntas gerais (tempo de atuação da empresa, quantidade de funcionários, cargo do respondente e região do país de atuação); ii) Bloco II - aspectos gerais (itens sobre reputação nomeado de aspectos gerais para não conduzir ao enviesamento das respostas); iii) Bloco III- características do desempenho econômico-financeiro. Ainda convém destacar que, entre as várias opções de escalas de verificações, optou-se pela escala do tipo Likert com sete pontos, partindo do "concordo totalmente" até o "discordo totalmente".

\subsection{Atividade de amostragem}

O levantamento de dados foi elaborado por e-mail usando o recurso do Google Forms. Uma pequena descrição apresentando o objetivo do trabalho foi encaminhada por e-mail com o pedido de colaboração para que os responsáveis/ gestores da empresa participassem da pesquisa.

Como o tamanho da população deste trabalho é desconhecido, e os membros poderiam ser identificados, optouse por uma amostragem por julgamento (BABBIE, 1999) em que foram considerados os guias e uma associação. A Tabela 1 apresenta a composição da amostra.

Tabela 1. Composição da amostra do estudo

\begin{tabular}{lc}
\hline \multicolumn{1}{c}{ Guias/ Associações/Prêmios } & Quantidade de empresas \\
\hline Associação Boa lembrança & 93 \\
Revista Prazeres da Mesa & 73 \\
Guia Quatro Rodas & 21 \\
Comer e Beber 2015-2018 & 27 \\
Guia folha Uol & 13 \\
Prêmio Paladar & 15 \\
\hline Total & $\mathbf{2 4 2}$ \\
\hline
\end{tabular}

Fonte: Elaborado pelos autores

Assim, o e-mail com o link de acesso ao questionário foi endereçado a 242 empreendimentos de todo o Brasil, conforme visto na Tabela 1, que atendessem aos seguintes critérios: i) ter mais de um ano de existência; ii) ter um chef de cozinha e iii) não ser uma rede de franquias. Deve-se destacar que São Paulo teve a maior quantidade de restaurantes por se tratar do primeiro polo gastronômico do país, segundo dados da Abrasel (2017), por isso guias locais foram considerados, a exemplo do Guia Folha Uol e do Prêmio Paladar.

Ao total, foram 68 questionários respondidos, no entanto dois questionários foram identificados como outliers por terem respostas tendenciosas e um questionário obteve notas até 6 , portanto, para não prejudicar as análises, optou-se pela exclusão, finalizando com 65 questionários válidos, sendo o índice de respostas de $26,8 \%$.

Vale destacar que todas as informações coletadas foram organizadas em uma base de dados eletrônica e analisadas utilizando algumas das ferramentas disponíveis no software estatístico Statical Package for the Social Science (SPSS 21.0), disponível no laboratório do Programa de Pós-Graduação em Administração da Universidade Federal de Pernambuco (PROPAD/UFPE). 


\subsection{Procedimentos de limpeza da escala}

Nesta etapa, discutiu-se, inicialmente, a análise descritiva geral dos itens e, em seguida, foram proferidas divisões para cada uma das dimensões.

\subsubsection{Análise descritiva geral}

A primeira verificação no conjunto dos itens consistiu na identificação de missing values. Nenhuma das variáveis apresentou dados faltantes, resultando em todas as respostas válidas. A fim de facilitar a apresentação de tais análises, foram adotados códigos para cada uma das variáveis, dispostos na primeira coluna da Tabela 2.

Embora a amostra considerasse restaurantes de todas as regiões brasileiras, com a maioria sendo de São Paulo, já que é o polo gastronômico do país, destaca-se que a maioria dos respondentes foi da região Nordeste $(50,80 \%)$, seguido da região Sudeste $(32,30 \%)$. Quando questionados sobre a quantidade de clientes atendidos por dia em média, apenas três empresas não responderam. Das empresas que responderam, $62,9 \%$ (39) atendem até 100 clientes. O valor do gasto médio por pessoa das empresas variou entre $R \$ 45,00$ a $R \$ 214,00$.

$\mathrm{Na}$ identificação das médias, na Tabela 2, a maioria das variáveis obtiveram médias altas, acima de seis, que indicam concordância quase totalmente dos respondentes. Essa informação parece coerente quando observado o tempo de atuação dessas empresas no mercado, já que a maioria $(61,6 \%)$ tem mais de dez anos.

Por outro lado, a variável REP 21, conforme apresentado na Tabela 1 , obteve média de 5,49, indicando concordância parcial sobre a indicação dos restaurantes em guias para uma boa imagem, devido ao fato de alguns restaurantes, provavelmente, não estarem em publicações ou não considerarem que essas publicações elevem o status do restaurante. Nesse caso, é uma variável que deve ser observada nos próximos testes.

Vale destacar que as variáveis referentes ao chef de cozinha obtiveram médias acima de cinco, indicando concordância parcial. No entanto, quando questionados sobre a possibilidade dos clientes estarem no restaurante por conta do chef, obteve-se uma média de 4,69 (REP20), revelando que, apesar do chef de cozinha dispor de uma boa imagem, não significa dizer que os clientes estejam ali por conta dele.

Tabela 2. Estatística descritiva do construto Reputação

\begin{tabular}{|c|c|c|c|}
\hline Variáveis & Itens da escala & Média & Desvio \\
\hline REP 1 & Os pratos e as bebidas servidos são sempre padronizados. & 6,12 & 1,097 \\
\hline REP 2 & O tempo de atendimento é considerado satisfatório. & 6,18 & 0,808 \\
\hline REP 3 & Os garçons são sempre corteses e educados. & 6,43 & 0,661 \\
\hline REP 4 & Os garçons têm uma boa apresentação pessoal (educação, aparência). & 6,46 & 0,709 \\
\hline REP 5 & O restaurante sempre se preocupa em atestar a qualidade do produto para o consumo. & 6,83 & 0,453 \\
\hline REP 6 & Raramente ocorrem erros (ex: pedidos, conta). & 5,80 & 0,922 \\
\hline REP 7 & Os banheiros estão sempre apropriados para uso. & 6,63 & 0,627 \\
\hline REP 8 & O restaurante alinha a arquitetura da casa com a proposta oferecida. & 6,74 & 0,509 \\
\hline REP 9 & As postagens nas redes sociais (Ex: Facebook, Instagram etc.) do restaurante são frequentes. & 6,22 & 1,281 \\
\hline REP 10 & O cardápio físico reflete o conceito do restaurante. & 6,69 & 0,683 \\
\hline REP 11 & Os garçons conhecem bem o cardápio (ingredientes / quantidade). & 6,38 & 0,823 \\
\hline REP 12 & O restaurante sempre mantém a limpeza (salão/ mesa/ talheres/copos/parte externa). & 6,66 & 0,594 \\
\hline REP 13 & O restaurante preocupa-se com a coerência entre as postagens nas redes sociais e a proposta gastronômica. & 6,71 & 0,522 \\
\hline REP 14 & O restaurante é bem avaliado na internet (Facebook, Google, Trip Advisor, Foursquare etc.). & 6,60 & 0,524 \\
\hline REP 15 & O restaurante tem um chef bastante reconhecido nas mídias em geral. & 5,52 & 1,659 \\
\hline REP 16 & A especialidade da casa é reconhecida pelos clientes. & 6,71 & 0,579 \\
\hline REP 17 & O chef tem fama difundida por ter recebido prêmios. & 5,12 & 1,850 \\
\hline REP 18 & O restaurante busca apoiar os pequenos produtores (ex: comprar produtos) & 5,29 & 1,637 \\
\hline REP 19 & Geralmente respondem-se, de imediato, às dúvidas/ reclamações do consumidor na internet. & 6,28 & 1,023 \\
\hline REP 20 & Muitos clientes vão ao restaurante por conta do chef. & 4,69 & 1,992 \\
\hline REP 21 & $\begin{array}{l}\text { A indicação em publicações da área (Revista Veja, Gula, Prazeres da Mesa etc.) tem valorizado a marca } \\
\text { deste restaurante. }\end{array}$ & 5,49 & 1,804 \\
\hline REP 22 & O restaurante sempre se preocupa com o descarte do lixo (ex: óleo, coleta seletiva). & 6,54 & 0,792 \\
\hline
\end{tabular}

Fonte: Dados da pesquisa 
Tabela 2. Estatística descritiva do construto Reputação (continuação)

\begin{tabular}{|c|c|c|c|}
\hline REP 23 & Os comentários no Trip Advisor em geral são positivos. & 6,37 & 0,698 \\
\hline REP 24 & No geral, o ambiente é muito confortável (temperatura /som/ ruídos/ mobiliário/iluminação). & 6,38 & 0,678 \\
\hline REP 25 & Os clientes geralmente indicam o restaurante para outras pessoas. & 6,63 & 0,517 \\
\hline REP 26 & As marcas dos produtos utilizadas são de alta qualidade. & 6,77 & 0,493 \\
\hline REP 27 & O restaurante preocupa-se em divulgar ações sociais que realiza. & 5,09 & 1,809 \\
\hline REP 28 & O restaurante procura fornecedores eticamente corretos. & 6,29 & 1,284 \\
\hline REP 29 & O restaurante preocupa-se com a apresentação dos pratos. & 6,69 & 0,635 \\
\hline REP 30 & Os pratos normalmente são servidos na temperatura adequada. & 6,72 & 0,545 \\
\hline REP 31 & O chef preocupa-se em colaborar/participar em eventos importantes do setor. & 5,83 & 1,420 \\
\hline REP 32 & O restaurante é um dos melhores em sua categoria. & 6,66 & 0,619 \\
\hline REP 33 & O restaurante, nos últimos anos, tem ganhado premiações. & 6,22 & 1,269 \\
\hline REP 34 & O restaurante preocupa-se em apoiar causas nobres. & 5,83 & 1,257 \\
\hline
\end{tabular}

Fonte: Dados da pesquisa

Uma convenção adotada nesta pesquisa para a análise do desvio padrão é a de que valores até 0,6 são indicativos de baixa dispersão; valores de 0,6 a 2 são indicativos de dispersão moderada e, acima de 2 , de elevada dispersão. Assim, as variáveis REP 5, REP 8, REP 12 REP 13, REP 14 REP 16, REP 25, REP 26 e REP 30 obtiveram dispersão muito baixa, as demais obtiveram dispersões moderadas. Segundo Costa (2011), variáveis com dispersões baixas informam muito pouco sobre o construto, podendo ser excluídas. No entanto resolveu-se analisar mais testes por cada dimensão para verificar, de fato, a necessidade de excluir tais variáveis.

\subsubsection{Dimensão qualidade do produto}

Para apresentação dos dados foi realizada a análise de correlação e consistência interna e, por último, a análise fatorial. Cada uma das etapas serviu de evidência para a seleção de quais variáveis estariam habilitadas para a formação da escala.

\subsubsection{Análise de correlação e consistência interna}

Segundo Costa (2011), os itens devem ter correlação mínima de 0,2 e estatisticamente não nula. Se menor que esse valor, significa que os itens não estão variando bem em conjunto. $O$ autor ainda aponta que a correlação não deve ser maior que 0,9 , pois indica colinearidade, portanto, recomenda-se a exclusão. Assim, após observar as correlações, verificou-se que a variável REP 1 não obteve correlação mínima de 0,2 com as demais, e a variável REP 5 só obteve uma boa medida de correlação com a variável REP 26, o que aponta que as duas podem ser variáveis problemáticas para essa dimensão.

$\mathrm{Na}$ análise de consistência interna, observou-se o valor do coeficiente alfa de Cronbach, obtendo-se o valor de 0,577, ou seja, um valor inaceitável, já que uma confiabilidade ao menos regular seria entre 0,6 e 0,7, e acima de 0,7 é considerada boa (COSTA, 2011). Outros autores, como Hair et al. (2005), apontam 0,6 como um valor aceitável para pesquisas como esta. Como as variáveis REP 1 e REP 5 não estavam se correlacionando bem com as demais, sugerindo que poderiam estar deflacionando o alfa, optou-se por excluí-las e realizar uma nova rodada, em que se obteve um alfa de 0,768 , confirmando uma melhoria da consistência dos itens da dimensão. A exclusão das variáveis, no entanto, só será realizada após verificações da análise fatorial e análise do conteúdo do item.

\subsubsection{Análise fatorial}

Essa etapa teve como objetivo verificar as cargas fatoriais, bem como a adequação dos itens na dimensão, para verificação da necessidade de exclusão de algum item.

O primeiro procedimento de análise realizado foi a verificação dos resultados do teste de esferecidade de Bartlett e o teste Kaiser-Meyer-Olkin (KMO), que são utilizados como indicativos de adequação da amostra de itens para a aplicação do modelo de análise fatorial. O teste $\mathrm{KMO}$, que mede o grau de correlação entre as variáveis, varia de 0 a 1, quanto mais próximo de 1, melhor. O critério mínimo adequado para o KMO é 0,5, segundo Hair et al (2005). Para o cálculo dos escores fatoriais fora utilizado o método dos componentes principais.

$\mathrm{O}$ resultado do teste com os cinco itens para o KMO foi 0,611 e o teste de esfericidade de Bartlett apresentou significância estatística do valor do qui-quadrado $\left(X^{2}=83,501, g l=10\right)$ a $p<0,001$, sendo considerados aceitos e, assim, apontando para a adequação da análise fatorial. Logo, foram tomadas as decisões para a extração dos fatores:

- Método dos componentes principais; 
- Critério de autovalores maiores que 1 (um) para definição da quantidade de fatores;

- Rotação ortogonal pelo método varimax.

Na primeira extração foram gerados dois fatores, o que contrariou a expectativa inicial de que haveria um único fator subjacente. Nessa extração, o primeiro fator explicava $46,39 \%$ e, o segundo, $21,05 \%$ da variância total. 0 resultado das cargas fatoriais dos cinco itens apontou que a variável REP 1 obteve carga fatorial de 0,361, apontando, mais uma vez, como uma candidata a exclusão. As variáveis REP 26 e REP 5 ficaram agrupadas no segundo fator.

Antes de excluir qualquer variável foram realizadas outras análises com as demais dimensões para verificar se os itens REP 26 e REP 5, que estavam aparecendo em outro fator, poderia se encaixar em outra dimensão. $O$ resultado de fato confirmou que os itens não se encaixavam em outras dimensões, no entanto a variável REP 16 (A especialidade da casa é reconhecida pelos clientes), abordada prioritariamente como uma variável da Dimensão Sucesso, surgiu como uma variável pertencente a essa dimensão, o que parece fazer sentido já que a dimensão busca medir qualidade do produto.

Além disso, ao analisar o conteúdo da variável REP 5 observou-se que o item conduzia o respondente a fornecer respostas altas. Isso é comprovado ao observar que o desvio padrão foi baixo, portanto, optou-se pela exclusão.

Dessa forma, foi realizada uma nova rodada da análise fatorial excluindo as variáveis REP 1 e REP 5 , e acrescentando a REP 16. O resultado do teste com os quatro itens (REP 26, REP 29, REP 30, REP 16) para o KMO foi 0,772 , e o teste de esfericidade de Bartlett apresentou significância estatística do valor do qui-quadrado $\left(X^{2}=74,972\right.$, $\mathrm{gl}=6$ ) a $\mathrm{p}<0,001$, sendo considerados aceitos $\mathrm{e}$, assim, apontando para a adequação da análise fatorial para o tratamento dos dados. O resultado da extração de autovalores indicou apenas um fator, como já se esperava, com variância total explicado de $62,4 \%$. Considera-se, portanto, que é possível seguir com os itens para a próxima fase. O valor final da consistência interna para o conjunto dos quatro itens foi de 0,794 , um valor satisfatório. Veja a tabela a seguir.

Tabela 3. Matriz de escores e comunalidade da Dimensão Qualidade do produto

\begin{tabular}{clcc}
\hline \multicolumn{1}{c}{ Cód. } & \multicolumn{1}{c}{ Itens } & Escore & Comu \\
\hline REP 16 & A especialidade da casa é reconhecida pelos clientes. & 0,751 & 0,563 \\
REP 26 & As marcas dos produtos utilizadas são de alta qualidade & 0,814 & 0,814 \\
REP 29 & O restaurante preocupa-se com a apresentação dos pratos. & 0,781 & 0,611 \\
REP 30 & Os pratos normalmente são servidos na temperatura adequada. & 0,814 & 0,663 \\
\hline
\end{tabular}

Fonte: Dados da pesquisa

\subsubsection{Dimensão Qualidade do atendimento}

A mesma sequência de análises estatísticas utilizadas na dimensão anterior foi utilizada nesta dimensão, bem como os critérios para a análise fatorial.

\subsubsection{Análise de correlação e consistência}

Ao analisar a correlação entre os itens, todos obtiveram correlação significativa acima de 0,2 com os demais, não apontando necessidade de exclusão de nenhum dos itens.

Na análise de consistência interna, observou-se o valor do alfa de Cronbach, obtendo-se o valor de 0,783 , um valor aceitável, confirmando mais uma vez que não há necessidade de excluir nenhum item.

\subsubsection{Análise fatorial}

O resultado do teste com os cinco itens para o KMO foi 0,764 , e o teste de esfericidade de Bartlett apresentou significância estatística do valor do qui-quadrado $\left(x^{2}=93,617, g l=10\right)$ a $p<0,001$, sendo considerados aceitos e, assim, apontando para a adequação da análise fatorial para o tratamento dos dados.

Utilizando os mesmos métodos da dimensão anterior foi gerado apenas um fator, com variância total de 55,05\%, que pode ser considerado aceitável por ser exploratório.

O resultado das cargas fatoriais dos cinco itens apontou que todas as variáveis apresentam cargas acima de 0,5 , o mínimo considerado neste trabalho. Além disso, ao se realizar uma fatorial com os demais itens, verificou-se que os itens dessa dimensão permaneceram agrupados, conferindo o ajuste entre eles mais uma vez. No entanto, ao se analisar o conteúdo dos itens, observou-se que REP 3 e REP 4 estavam similares. Observando as médias, constata-se uma aproximação, assim optou-se pela exclusão do item com menor escore e menor comunalidade. Nesse caso, o item REP 4.

Após a exclusão, o resultado do teste com os quatro itens para o KMO foi 0,711 e o teste de esfericidade de Bartlett apresentou significância estatística do valor do qui-quadrado $\left(X^{2}=59,874, \mathrm{gl}=6\right)$ a $p<0,001$, sendo considerados aceitos e, assim, apontando para a adequação da análise fatorial para o tratamento dos dados. Foi gerado apenas 
um fator com variância total de $57,56 \%$. O valor final da consistência interna para o conjunto dos quatro itens foi de 0,743 , que representa uma medida satisfatória.

Tabela 4. Matriz de escores e comunalidade da Dimensão Qualidade do atendimento

\begin{tabular}{clcc}
\hline Cód. & \multicolumn{1}{c}{ Itens } & Escore & Comu \\
\hline REP 2 & O tempo de atendimento é considerado satisfatório. & 0,727 & 0,529 \\
REP 3 & Os garçons são sempre corteses e educados. & 0,810 & 0,656 \\
REP 6 & Raramente ocorrem erros (ex: pedidos, conta). & 0,719 & 0,516 \\
REP 11 & Os garçons conhecem bem o cardápio (ingredientes/quantidade). & 0,776 & 0,656 \\
\hline
\end{tabular}

Fonte: Dados da pesquisa

\subsubsection{Dimensão Qualidade do ambiente}

A mesma sequência de análises estatísticas utilizadas na dimensão anterior foi utilizada nesta dimensão, bem como os critérios para análise fatorial.

\subsubsection{Análise de correlação e consistência}

Ao analisar a correlação, observou-se que a variável REP 8 obteve baixa correlação com as variáveis REP 10 e REP 12, apontando possivelmente a necessidade de excluí-la. O valor do alfa de Cronbach foi de 0,732 na análise de consistência interna. Após a exclusão da variável REP 8 , o novo alfa foi de 0,761 , porém optou-se por fazer essa exclusão após verificar as próximas análises.

\subsubsection{Análise fatorial}

Utilizando os mesmos métodos anteriores foi gerado um fator com variância total de $49,25 \%$, considerado aceitável por estar muito próximo do ideal.

$\mathrm{O}$ resultado do teste com os cinco itens para o $\mathrm{KMO}$ foi 0,699 e o teste de esfericidade de Bartlett apresentou significância estatística do valor do qui-quadrado $\left(x^{2}=78,212, g l=10\right)$ a $p<0,001$, sendo considerados aceitos e assim apontando para a adequação da análise fatorial.

O resultado das cargas fatoriais dos cinco itens apontou que todas as variáveis apresentam cargas acima de 0,5 , exceto a variável REP 8 , com valor de 0,389 , além da baixa comunalidade, confirmando mais uma vez a necessidade de exclusão.

A verificação do sentido dos itens indicou que a exclusão do item REP 8 não provocaria perda no sentido da dimensão, já que está tratando mais de coerência de design do que realmente um ambiente de qualidade.

A REP 7 (Os banheiros estão sempre apropriados para o uso) apresentou baixa comunalidade e ao analisar as demais variáveis observou-se que o seu conteúdo estava similar à variável REP 12 (O restaurante sempre mantém limpeza: salão/mesa/utensílios/parte externa), portanto poderia incluí-la com uma readequação do item, e assim justifica a exclusão da REP 7. Dessa forma após uma nova rodada excluindo REP 7 e REP 8, o KMO foi de 0,698 e o teste de esfericidade de Bartlett apresentou significância estatística do valor do qui-quadrado $\left(X^{2}=56,791, \mathrm{gl}=3\right)$ a $p<0,001$, sendo considerados aceitos e assim apontando para a adequação da análise fatorial para o tratamento dos dados. Foi gerado apenas um fator com variância total de $70,67 \%$. $O$ valor final da consistência interna para o conjunto dos 3 itens foi de 0,789 que representa uma boa consistência.

Tabela 5. Matriz de escores e comunalidade da dimensão Qualidade do ambiente

\begin{tabular}{|c|c|c|c|}
\hline Cód. & Itens & Escore & Comu \\
\hline REP 10 & O cardápio físico reflete o conceito do restaurante. & 0,859 & 0,733 \\
\hline REP 12 & O restaurante sempre mantém limpeza (salão/ mesa/utensílios/parte externa). & 0,856 & 0,738 \\
\hline REP 24 & No geral o ambiente é muito confortável (temperatura /som/ ruídos/ mobiliário/iluminação). & 0,806 & 0,648 \\
\hline
\end{tabular}

Fonte: Dados da pesquisa

\subsubsection{Dimensão Força das mídias}

A mesma sequência de análises estatísticas utilizadas na dimensão anterior foi utilizada nesta dimensão, bem como os critérios para análise fatorial. 


\subsubsection{Análise de correlação e consistência}

Após análise de correlação as variáveis REP13 e REP19 apresentaram baixa correlação com as demais, portanto candidatas à exclusão.

$\mathrm{Na}$ análise de consistência interna o valor do alfa de Cronbach foi de 0,559 , valor não aceitável. Assim, observou a exclusão primeiramente da variável REP 13 , porém o alfa diminuiu para 0,55 , logo se resolveu recolocar a variável e excluir a variável REP 19 que elevou o alfa para 0,578. Portanto, a única variável possível de exclusão seria a REP 19, mas mesmo assim os itens não parecem confiáveis para mensuração do construto. Com isso, optou-se por executar a análise fatorial para verificar melhor os dados.

\subsubsection{Análise fatorial}

Utilizando os mesmos métodos anteriores foram gerados dois fatores, o primeiro fator com variância de $41,87 \%$ e o segundo com variância e $21,89 \%$. O segundo fator é composto apenas pelas variáveis REP 19 que não estava se correlacionando bem com as demais variáveis, conforme já discutido anteriormente.

O resultado do teste com os cinco itens para o KMO foi 0,591 e o teste de esfericidade de Bartlett apresentou significância estatística do valor do qui-quadrado $\left(x^{2}=58,593, g l=10\right)$ a $p<0,001$, sendo considerados aceitos e, assim, apontando para a adequação da análise fatorial para o tratamento dos dados. O resultado das cargas fatoriais dos cinco itens apontou que todas as variáveis apresentaram cargas acima de 0,5.

Ao verificar o conteúdo do item REP 19 (geralmente responde-se, de imediato, às dúvidas/reclamações do consumidor na internet), observou-se que não estava contemplando a dimensão, pois o item tratava da agilidade em responder aos clientes. Além disso, a variável não se correlacionou bem no fator, justificando-se a exclusão. Analisou-se, ainda, o conteúdo da variável REP 13 já que apresentou baixa correlação se correlacionando satisfatoriamente com duas das variáveis, e o desvio padrão foi muito baixo, assim, concluiu-se que a exclusão do item não prejudicaria o sentido da dimensão.

Após estas exclusões uma nova rodada foi gerada e KMO foi 0,558 e o teste de esfericidade de Bartlett apresentou significância estatística do valor do qui-quadrado $\left(x^{2}=45,344, g l=10\right)$ a $p<0,001$, sendo considerados aceitos e assim apontando para a adequação da análise fatorial para o tratamento dos dados. Foi gerado apenas um fator com variância total de $63,30 \%$. O resultado das cargas fatoriais dos três itens apontou que todas as variáveis apresentaram cargas acima de 0,5 . O valor final da consistência interna para o conjunto dos 3 itens foi de 0,577 que por estar próximo do valor mínimo, mas aceito por se tratar de uma pesquisa exploratória.

Tabela 6. Matriz de escores e comunalidade da Dimensão Força das mídias

\begin{tabular}{clcc}
\hline Cód. & \multicolumn{1}{c}{ Itens } & Escore & Comu \\
\hline REP 9 & As postagens nas redes sociais (Ex: Facebook, Instagram etc.) do restaurante são frequentes. & 0,671 & 0,450 \\
REP 14 & O restaurante é bem avaliado na internet (Facebook, Google, Trip Advisor, Foursquare etc.). & 0,897 & 0,805 \\
REP 23 & Os comentários no Trip Advisor em geral são positivos. & 0,803 & 0,644 \\
\hline
\end{tabular}

Fonte: Dados da pesquisa

\subsubsection{Dimensão Imagem do chef}

A mesma sequência de análises estatísticas utilizadas na dimensão anterior foi realizada nesta dimensão, bem como os critérios para análise fatorial.

\subsubsection{Análise de correlação e consistência}

A análise de correlação comprovou que todas as variáveis obtiveram correlação adequada entre si. Na análise de consistência interna observou-se o valor do alfa de Cronbach, de 0,807 o que é bom para a representatividade da consistência dos itens.

\subsubsection{Análise fatorial}

Utilizando os mesmos métodos anteriores foi gerado um único fator com a variância total de $65,95 \%$. O resultado das cargas fatoriais dos cinco itens apontou que todas as variáveis apresentaram cargas acima de 0,5 , não sendo necessária a exclusão de nenhum item.

O resultado do teste com os cinco itens para o KMO foi 0,711 e o teste de esfericidade de Bartlett apresentou significância estatística do valor do qui-quadrado $\left(x^{2}=128,223, g l=6\right)$ a $p<0,001$, sendo considerados aceitos e assim apontando para a adequação da análise fatorial para o tratamento dos dados. 
Tabela 7. Matriz de escores e comunalidade da dimensão Imagem do chef

\begin{tabular}{clcc}
\hline Cód. & \multicolumn{1}{c}{ Itens } & Escore & Comu \\
\hline REP 15 & O restaurante tem um chef bastante reconhecido nas mídias em geral. & 0,910 & 0,827 \\
REP 17 & O chef tem fama difundida por ter recebido prêmios. & 0,905 & 0,819 \\
REP 20 & Muitos clientes vão ao restaurante por conta do chef. & 0,601 & 0,361 \\
REP 31 & O chef preocupa-se em colaborar/participar em eventos importantes do setor. & 0,794 & 0,631 \\
\hline
\end{tabular}

Fonte: Dados da pesquisa

\subsubsection{Dimensão Sucesso}

A mesma sequência de análises estatísticas utilizadas na dimensão anterior foi utilizada nesta dimensão, bem como os critérios para análise fatorial.

\subsubsection{Análise de correlação e consistência}

A análise de correlação apresentou que apenas a variável REP 21 não obteve correlação adequada sendo uma possível candidata a exclusão. $\mathrm{Na}$ análise de consistência interna o valor do alfa de Cronbach foi de 0,577 e assim após a exclusão da variável REP 21 , o novo valor do alfa foi de 0,676 , considerado mais adequado. No entanto a exclusão da variável será ou não realizada após analisar o conteúdo do item e os escores fatoriais.

\subsubsection{Análise fatorial}

Utilizando os mesmos métodos anteriores foi gerado um único fator com variância total de $48,70 \%$ que é um valor baixo, mas como é próximo de $50 \%$ e por ser uma pesquisa exploratória pode ser considerado. O resultado do teste com os 5 itens para o KMO foi 0,604 e o teste de esfericidade de Bartlett apresentou significância estatística do valor do qui-quadrado $\left(X^{2}=85,061, \mathrm{gl}=10\right)$ a $p<0,001$, sendo considerados aceitos. Todas as variáveis apresentaram cargas fatoriais acima de 0,5 , exceto a variável REP 21 , com carga de 0,410 , além de que ao analisar o conteúdo percebe-se que a exclusão da mesma não prejudica o sentido da dimensão.

Conforme já foi apontada a variável REP 16 apresentou-se melhor agrupada na Dimensão Qualidade do produto e analisando seu conteúdo não houve perda de sentido da dimensão aqui analisada, portanto optou-se por excluí-la.

Após a exclusão das variáveis REP 16 e REP 21 uma nova rodada gerou apenas um fator conforme já esperava com variância total de $68,89 \%$. O resultado do teste com os 3 itens para o KMO foi 0,666 e o teste de esfericidade de Bartlett apresentou significância estatística do valor do qui-quadrado $\left(x^{2}=53,498, \mathrm{gl}=3\right)$ a $p<0,001$, sendo considerados aceitos e melhores do que a primeira rodada, apontando a adequação da análise fatorial para o tratamento dos dados. $O$ resultado das cargas fatoriais dos itens bem como a comunalidade encontra-se a seguir na Tabela. $O$ valor do alfa de Cronbach foi de 0,685 que representa um valor adequado.

Tabela 8. Matriz de escores e comunalidade da Dimensão Sucesso

\begin{tabular}{clcc}
\hline Cód. & \multicolumn{1}{c}{ Itens } & Escore & Comu \\
\hline REP 25 & Os clientes geralmente indicam o restaurante para outras pessoas. & 0,775 & 0,600 \\
REP 32 & O restaurante é um dos melhores em sua categoria. & 0,878 & 0,772 \\
REP 33 & O restaurante nos últimos anos tem ganhado premiações. & 0,834 & 0,695 \\
\hline
\end{tabular}

Fonte: Dados da pesquisa

\subsubsection{Dimensão Responsabilidade socioambiental}

A mesma sequência de análises estatísticas utilizadas na dimensão anterior foi utilizada nesta dimensão, bem como os critérios para análise fatorial.

\subsubsection{Análise de correlação e consistência}

Após análise de correlação é possível constatar que as variáveis REP 22 e REP 28 são possíveis de serem excluídas já que não obtiveram correlação adequada com as outras variáveis. O valor do alfa de Cronbach foi de 0,629 , nesse caso optou-se por excluir a variável REP 22 e gerou uma nova rodada que resultou no alfa de 0,643 que pode ser considerado adequado, no entanto será analisado o sentido do item para efetuar a exclusão. 


\subsubsection{Análise fatorial}

Utilizando os mesmos métodos anteriores foi gerado um único fator com variância total de $41,27 \%$, que é considerado um valor muito baixo. O resultado do teste com os cinco itens para o KMO foi 0,645 e o teste de esfericidade de Bartlett apresentou significância estatística do valor do qui-quadrado $\left(X^{2}=41,766, g l=10\right)$ a $p<0,001$, sendo considerados aceitos. As cargas fatoriais dos cinco itens foram acima de 0,5 , exceto a variável REP 22 , com carga de 0,397 , confirmando mais uma vez a necessidade de sua exclusão.

Ao analisar o conteúdo do item REP 22, observou-se que o item estava relacionado a questões ambientais, enquanto os demais estavam ligados a questões sociais, o que se confirma quando analisado a correlação entre os itens. Assim, os itens REP 28 e REP 22 foram excluídos e a dimensão renomeada para Responsabilidade social.

Após a exclusão dos itens uma nova rodada gerou apenas um fator conforme já esperava com variância total de $57,45 \%$ considerado adequado. O resultado do teste com os três itens para o KMO foi 0,631 e o teste de esfericidade de Bartlett apresentou significância estatística do valor do qui-quadrado $\left(x^{2}=23,115, g l=3\right)$ a $p<0,001$, sendo considerados aceitos apontando a adequação da análise fatorial para o tratamento dos dados. $O$ resultado das cargas fatoriais dos itens, bem como a comunalidade encontra-se na Tabela 8. Na análise de consistência interna, observou-se que o valor do alfa de Cronbach, de 0,613 representa um valor adequado.

Tabela 9. Matriz de escores e comunalidade da Dimensão Responsabilidade social

\begin{tabular}{clcc}
\hline Cód. & Itens & Escore & Comu \\
\hline REP 18 & O restaurante busca apoiar os pequenos produtores (ex: comprar produtos etc.). & 0,691 & 0,477 \\
REP 27 & O restaurante preocupa-se em divulgar ações sociais que realiza. & 0,787 & 0,620 \\
REP 34 & O restaurante preocupa-se em apoiar causas nobres. & 0,792 & 0,627 \\
\hline
\end{tabular}

Fonte: Dados da pesquisa

\subsection{Análise de confiabilidade e validade}

Para o cumprimento deste passo, procedeu-se inicialmente a validade de translação (validade de face e conteúdo) que foi feita inicialmente no passo dois deste roteiro. Recomenda-se, no entanto, que fossem verificados novamente todos os itens que restaram para fortalecer a evidência dessa validade (COSTA, 2011). Dessa forma, constatou-se que as variáveis REP 3, REP 12 precisavam ser reescritas, conforme o Quadro 2.

Quadro 2. Ajustes dos itens de Reputação

\begin{tabular}{|c|c|c|}
\hline Cód. & Item original & Item reescrito \\
\hline \multicolumn{3}{|c|}{ Qualidade do atendimento } \\
\hline REP 3 & Os garçons são sempre corteses e educados. & $\begin{array}{c}\text { Os garçons deste restaurante sempre estão bem } \\
\text { apresentados (educados, boa aparência e corteses) }\end{array}$ \\
\hline \multicolumn{3}{|c|}{ Qualidade do ambiente } \\
\hline REP 12 & O restaurante sempre mantém limpeza (salão/ mesa/ & O restaurante sempre mantém limpeza (salão/ \\
utensilios/parte externa).
\end{tabular}

Fonte: Dados da pesquisa

Para verificação da confiabilidade, observou-se os valores de alfa de Cronbach referente a cada uma das dimensões que são apresentados na Tabela 10. Enquanto que para análise da validade convergente fora observada as medidas nos escores fatoriais de cada dimensão dos construtos que indicaram evidência de validade com as cargas acima de 0,4 . 
Tabela 10. Resumo das medidas das dimensões de Reputação

\begin{tabular}{lcccc}
\hline \multicolumn{1}{c}{ Dimensão } & Média final & Desvio final & Variância explicada & Alfa de Cronbach \\
\hline Qualidade do produto & 6,72 & 0,53 & $62,40 \%$ & 0,794 \\
Qualidade do atendimento & 6,25 & 0,78 & $55,05 \%$ & 0,783 \\
Qualidade do ambiente & 6,56 & 0,65 & $70,67 \%$ & 0,789 \\
Força das mídias & 6,38 & 0,83 & $63,30 \%$ & 0,577 \\
Imagem do chef & 5,29 & 1,73 & $65,95 \%$ & 0,807 \\
Sucesso & 6,50 & 0,80 & $68,89 \%$ & 0,685 \\
Responsabilidade social & 5,40 & 1,56 & $57,45 \%$ & 0,613 \\
\hline
\end{tabular}

Fonte: Dados da pesquisa

\subsection{Normas e recomendação de usos}

Algumas recomendações são básicas para a construção de qualquer escala, tais como: i) Os itens devem ser dispostos de forma aleatória de preferência em blocos; ii) incluir itens para a validade de critério, isto é, com atenção e cuidado para não prejudicar a qualidade das informações e nem gerar indisposição do respondente; ii) verificar as possibilidades de variação do número de pontos, observando a capacidade cognitiva do respondente, além de que, quando a aplicação é realizada online, observar que a visualização de todos os pontos requer mais atenção do respondente. Acredita-se que manter a escala original ajuda na interpretação dos resultados (COSTA, 2011).

Quanto às recomendações de uso, entende-se que a escala pode ser utilizada para avaliar o potencial da reputação na empresa, contribuindo para entender quais variações e fatores interferem no aumento desse potencial. Lembrando que os respondentes da escala devem ser os gestores das empresas. No caso da aplicação da escala para restaurantes ou serviços em geral sem a figura do chef de cozinha, recomenda-se excluir os itens referentes a ele, além de outras adaptações, se julgarem necessário.

\section{Considerações Finais}

O tema deste trabalho se originou do interesse em analisar a reputação, um recurso intangível, fonte de vantagem competitiva para os diversos segmentos, no caso específico para restaurantes de alta gastronomia. A pesquisa se classifica como metodológica e descritiva, tendo em vista que se propõe à elaboração de instrumentos de coleta de dados, bem como à descrição do construto em análise.

Com a construção da escala, buscou-se trazer contribuições teóricas e empíricas para a área de estratégia bem como ao setor em estudo. A contribuição teórica deste trabalho consiste principalmente na construção da escala de reputação. Em uma perspectiva mais ampla e conceitual, ao integrar temáticas, como recursos intangíveis no setor de restaurantes, foi possível propor um novo olhar para compreender o comportamento de um recurso intangível em um contexto carente como o de restaurantes. Essa perspectiva abre novas possibilidades para avançar na compreensão e melhoria da reputação e atuação de restaurantes.

Na parte prática, a escala, representa uma ferramenta de diagnóstico organizacional para intervenções em diferentes contextos. Em outras palavras, o instrumento pode interessar aos gestores que precisam obter diagnósticos objetivos acerca do potencial de seus recursos, bem como a pesquisadores para estudos voltados para o setor de hospitalidade, além de diversos outros serviços com o cuidado da adequação da escala.

Para condução deste estudo utilizou-se a recomendação de DeVellis (1991) que entende que uma amostragem seria suficiente, porém, outros autores recomendam utilizar pelo menos duas amostragens (CHURCHILL, 1979; COSTA, 2011) para validar a escala. Portanto, a ausência de uma segunda amostragem pode ser considerada uma das limitações do trabalho. Assim, sugerem-se novas validações, utilizando uma segunda amostragem com maior número de respostas válidas, evitando problemas com o uso das técnicas estatísticas que são limitadas ao tamanho da amostra.

Além disso, este trabalho concentrou-se em mensurar a reputação apenas sob o ponto de vista de um único gestor/ proprietários, não considerando a visão de outros gestores. Assim, assume-se que esta é uma visão parcial do fenômeno estudado.

Por fim, ressalta-se que a escala desenvolvida pode também ser relacionada com vários fenômenos organizacionais, como por exemplo, o desempenho, a internacionalização das empresas, processo de transformação em franquias etc. 


\section{Referências}

ASSOCIAÇÃO BRASILEIRA DE BARES E RESTAURANTES - ABRASEL. Perfil da Abrasel. [2017]. Disponível em:<https://abrasel.com.br/abrasel/perfil-da-abrasel/>. Acesso em: 12 Jan. 2018.

ARAGÃO, F. B. P. et al. Curtiu, comentou, comprou. A mídia social digital Instagram e o consumo. Revista Ciências Administrativas, Fortaleza, v. 22, n. 1, p. 130-161, jan./jun. 2016.

AZEVEDO, T. R. et al. Avaliação da qualidade percebida de um serviço por meio de site de redes sociais. Revista Ciências Administrativas, Fortaleza, v. 22, n. 1, p. 162-191, jan./jun. 2016.

BARNEY, J. Firm resources and sustained competitive advantage. Journal of Management, [s. I.], v. 17, n. 1, p. 99-120, 1991.

CHURCHILL JR, G. A. A paradigm for developing better measures of marketing constructs. Journal of Marketing Research, [s. I.], v. 16, n. 1, p. 64-73, 1979.

COSTA, F. J. Mensuração e desenvolvimento de escalas: aplicações em administração. Rio de Janeiro: Ciência Moderna, 2011.

DIERICKX, I.; COOL, K. Asset stock accumulation and sustainability of competitive advantage. Management Science, [s. I.], v.35, n.12, p. 1504-1513, 1989.

FAVORETO, R. L.; VIEIRA, S. F. A Produção Internacional em RBV: características gerais e tratativa dos recursos na pesquisa em organizações de menor porte. Revista Ibero-Americana de Estratégia, São Paulo, v. 13, n. 2, p. 25, Abr./Jun. 2014.

FOMBRUN, C.J.; SHANLEY, M. What's in a name? Reputation building and corporate strategy. Academy of Management Journal, Briarcliff Manor, NY, v. 33, n. 2, p. 233-258, 1990.

FOMBRUN, C.J.; GARDBERG, N. A.; SEVER, J. M. The Reputation Quotient SM: a multi-stakeholder measure of corporate reputation. Journal of Brand Management, [s. I.], v. 7, n. 4, p. 241-255, 2000.

GOTSI, M.; WILSON, A.M. Corporate reputation: seeking a definition. Corporate Communications: an International Journal, [s. I.], v. 6, n. 1, p. 24-30, 2001.

GODFREY, P. C.; HILL, C. W. L. The problem of unobservables in strategic management research. Strategic Management Journal, [s. I.], v. 16, n. 7, p. 519-533, 1995.

HAl, J. F et al.. Fundamentos de métodos de pesquisa em administração. Porto Alegre: Bookman Editora, 2005.

HALL, R. The strategic analysis of intangible resources. Strategic Management Journal, [s. I.], v.13, n.2, p.135-144, Feb. 1992.

HALL, R. A framework linking intangible resources and capabilities to sustainable competitive advantage. Strategic Management Journal, [s. I.], v. 14, n. 8, p. 607-618, Nov. 1993.

HANEFORS, M.; MOSSBERG, L. Searching for the extraordinary meal experience. Journal of Business and Management, [s. I.], v. 9, n. 3, p. 249-270, 2003.

HELM, S. Designing a formative measure for corporate reputation. Corporate Reputation Review, [s. I.], v. 8, n. 2, p. 95-109, 2005.

MICHALISIN, M. D.; SMITH, R. D.; KLINE, D.M. In search of strategic assets. The International Journal of Organizational Analysis, [s. I.], v. 5, n. 4, p. 360-387, 1997.

ROSSITER, J. R. The C-OAR-SE procedure for scale development in marketing. International journal of research in marketing, [s. I.], v. 19, n. 4, p. 305-335, 2002. 
SALAZAR, V. S. Aquisição e desenvolvimento de recursos estratégicos de restaurantes gastronômicos: estudo de multicasos na América Latina. 2015. Tese (Doutorado em Administração) - Programa de Pós-Graduação em Administração, Universidade Federal de Pernambuco, Recife, 2015.

SMITH, A. D. Resource based view of the firm: measures of reputation among health service-sector businesses. Health marketing quarterly, [s. I.], v. 25, n. 4, p. 361-382, 2008.

SPECTOR, P. E. Summated rating scale construction: an introduction. Newbury Park, CA: Sage, 1992.

STIERAND, M; DÖRFLER, V. Reflecting on a phenomenological study of creativity and innovation in haute cuisine. International Journal of Contemporary Hospitality Management, [s. I.], v. 24, n. 6, p. 946-957, 2012.

THOMAZ, J. C.; BRITO, E. P. Z. Reputação corporativa: construtos formativos e implicações para a gestão. RACRevista de Administração Contemporânea, Curitiba, v. 14, n. 2, mar./abr. 2010.

WALKER, K. A systematic review of the corporate reputation literature: Definition, measurement, and theory. Corporate Reputation Review, [s. I.], v. 12, n. 4, p. 357-387, 2010.

\section{Contato:}

Luana de Oliveira Alves

E-mail: admluanaalves@gmail.com

Viviane Santos Salazar

E-mail: viviane_salazar@yahoo.com.br

Indira Gandhi Bezerra-de-Sousa

E-mail: indirabs16@gmail.com 